\title{
LOCAL AND GLOBAL EXTREMA FOR FUNCTIONS OF SEVERAL VARIABLES
}

\author{
BRUCE CALVERT and M. K. VAMANAMURTHY
}

(Received 18 April; revised 18 September 1979)

Communicated by J. B. Miller

\begin{abstract}
Let $p: \mathbf{R}^{2} \rightarrow \mathbf{R}$ be a polynomial with a local minimum at its only critical point. This must give a global minimum if the degree of $p$ is $<5$, but not necessarily if the degree is $\geqslant 5$. It is an open question what the result is for cubics and quartics in more variables, except cubics in three variables. Other sufficient conditions for a global minimum of a general function are given.
\end{abstract}

1980 Mathematics subject classification (Amer. Math. Soc.): 26 B 99, 26 C 99.

\section{Introduction}

In a course on calculus of several variables one has to deal with global extrema as well as local. If $f: \mathbf{R} \rightarrow \mathbf{R}$ has a local minimum at a certain point, and has no other critical points, then the local minimum becomes the global minimum. The purpose of this paper is to extend this result to functions of several variables. In general, the analogous result is false for several variables as shown in Theorem 1. However, the result holds under certain additional conditions. These are obtained in Theorems $2,3,4,5$ and 6.

For integers $n \geqslant 1$ we let $\mathbf{x}=\left(x_{1}, x_{2}, \ldots, x_{n}\right)$ denote a point in the Euclidean space $\mathbf{R}^{n}$, and $\|\mathbf{x}\|=\left(x_{1}^{2}+\ldots+x_{n}^{2}\right)^{\ddagger}$, the norm of $\mathbf{x}$. We denote the derivative of $f: \mathbf{R}^{n} \rightarrow \mathbf{R}$ by $D f=\left(D_{1} f, \ldots, D_{n} f\right)$. We also use the notations $(x, y)$ and $(x, y, z)$ to denote points in $\mathbf{R}^{2}$ and $\mathbf{R}^{3}$.

We make use of the notion of topological degree and refer the reader to Cronin (1964) for this concept.

We thank David B. Gauld for his comments. 


\section{A counterexample}

We give a simple counterexample in Theorem 1 to show that a unique critical point may give a local minimum but not a global minimum. In general for a nonpolynomial counterexample one may take almost any function $f: \mathbf{R}^{n} \rightarrow \mathbf{R}, n \geqslant 2$, with a local minimum at $\mathbf{a}$ and $f(\mathbf{b})<f(\mathbf{a})$ for some $\mathbf{b}$, and restrict $f$ to some set containing $\mathbf{a}$ and $\mathbf{b}$ but no critical points $\neq \mathbf{a}$ of $f$ and which is diffeomorphic to $\mathbf{R}^{n}$.

THEOREM 1. For all integers $p \geqslant 5$ and $q \geqslant 2$ there exists a polynomial in $\mathbf{x}=\left(x_{1}, \ldots, x_{q}\right)$ of degree $p$ with local minimum at the only critical point $\mathbf{0}$ but which is not bounded above or below.

ProOF. Let $f(\mathbf{x})=x_{1}^{2 m}\left(1+\sum_{i=2}^{q} x_{i}\right)^{3}+\sum_{i=2}^{q} x_{i}^{2 n}$, where $n, m \geqslant 1$, with

$$
\max (2 m+3,2 n)=p \text {. }
$$

For $\mathbf{x}$ near $\mathbf{0},\left(1+\sum_{i=2}^{q} x_{i}\right) \geqslant \frac{1}{2}$ and $f(\mathbf{x}) \geqslant x_{1}^{2 m} / 2+\sum_{i=2}^{q} x_{i}^{2 n}>0$ for $\mathbf{x} \neq \mathbf{0}$. For a critical point we have $D f(\mathbf{x})=\left(D_{1} f(\mathbf{x}), \ldots\right)=\mathbf{0}$. That is,

$$
D_{1} f(x)=2 m x_{1}^{2 m-1}\left(1+\sum_{i=2}^{q} x_{i}\right)^{3}=0
$$

and for $j=2, \ldots, q, D_{j} f(x)=3 x_{1}^{2 m}\left(1+\sum_{i=2}^{q} x_{i}\right)^{2}+2 n x_{j}^{2 n-1}=0$. From the second equation, if $x_{1}=0$ then $x_{j}=0$. From the first, if $x_{1} \neq 0$ then $1+\sum_{i=2}^{q} x_{i}=0$, and then from the second, $x_{j}=0$, for each $j=2, \ldots, q$, a contradiction. Hence 0 is the only critical point. If $2 m+3>2 n, f\left(x_{1}, x_{1}, 0, \ldots, 0\right)$ is of odd degree in $x_{1}$, and if $2 m+3<2 n$, then $f\left(x_{1}^{2 n}, x_{1}, 0, \ldots, 0\right)$ is of odd degree $4 m n+3$. In either case $f \rightarrow \pm \infty$ as $x_{1} \rightarrow \pm \infty$.

\section{Sufficient conditions}

From Theorem 1 we see that a unique critical point which gives a local minimum need not give a global minimum even for polynomials in two or more variables, provided the degree exceeds four. What about degree $\leqslant 4$ ? Of course, for degree one the result is trivially true, and for degree two the result follows by homogeneity or from the theory of quadratic forms. The result is also true for convex functions, and some functions derived from these. We now show the result is true for a cubic in two or three variables and a quartic in two variables. The remaining cases are still open. However, we note that Theorem 6 shows that if the result holds for a quartic in $n$ variables then it holds for a cubic in $n+1$ variables.

THEOREM 2. Let $f: \mathbf{R}^{n} \rightarrow \mathbf{R}$ be a $C^{1}$ function with a local minimum at $\mathbf{0}$ and no other critical point. Suppose that if $\mathbf{x}(t)$ is an integral curve for the gradient $D f$ of $f$ with $f(\mathbf{x}(t))$ bounded then $\mathbf{x}(t)$ is also bounded. Then $f$ has a global minimum at 0 . 
Proof. We may assume that $f(0)=0$. For $s>0$, small, we have a compact $(n-1)$-manifold $\{\mathbf{x}:\|x\|$ small, $f(\mathbf{x})=s\}=c(s)$. For $s$ and $x$ large possibly, we extend these level sets by continuity, in fact, if we parametrize $\mathrm{c}(s)$ as $\mathrm{c}(s, t)$, then $\partial \mathbf{c} / \partial s(s, \mathbf{t})=D f(\mathbf{c}(s, \mathbf{t})) /\|D f(\mathbf{c}(s, \mathbf{t}))\|^{2}$. We suppose there is $k=\sup \{s: \mathbf{c}(s)$ a compact $(n-1)$-manifold $\}<\infty$. If the images of $\mathbf{c}(s)$ for $s<k$ cover $\mathbf{R}^{n}$ we are done. Otherwise $\mathbf{c}(k)$ is unbounded, and there is an integral curve $\mathbf{x}(r)$ with $f(\mathbf{x}(r)) \rightarrow k$ as $r \rightarrow \infty$ and $\mathbf{x}(r)$ unbounded. This contradicts our hypothesis. Thus $k=\infty$ and hence the images of $\mathbf{c}(s)$ for $s<k$ cover $\mathbf{R}^{n}$.

COROllary 1. Let $f: \mathbf{R}^{n} \rightarrow \mathbf{R}$ be $C^{1}$ with a local minimum at $\mathbf{0}$ and no other critical point. Suppose there are $\delta>0$ and $M>0$ such that for all $x \in \mathbf{R}^{n}$ with $\|x\| \geqslant M$ we have $\|D f(\mathbf{x})\| \geqslant \delta$. Then $f$ has a global minimum at $\mathbf{0}$.

ProOF. This follows from the fact that if the integral curve $\mathbf{x}(s)$ is parametrized with respect to arc length $s$, then $d f / d s=\|D f(\mathbf{x}(s))\|$.

THEOREM 3. Let $p: \mathbf{R}^{n} \rightarrow \mathbf{R}$ be a polynomial of degree $q$. Let $p_{q}$, the homogeneous part of degree $q$, be such that after any change of coordinates there is a term in $x_{n}^{q}$ or $x_{n}^{q-1} x_{j}$ for some $j<n$. If $n=2$, this means there are no repeated linear factors in $p_{q}$. For $n>2$, this means if $p_{q}(\mathbf{x})=0$ when $P \mathbf{x}=\mathbf{0}, P$ a projection of rank $<n$, we do not have a function $\alpha$ of $(I-P) \mathbf{x}$ with $\left|p_{q}(\mathbf{x})\right| \leqslant\|P \mathbf{x}\|^{2} \alpha((I-P) \mathbf{x})$.

Suppose $p$ has a local minimum at $\mathbf{0}$ but no other critical point. Then $p$ has a global minimum at $\mathbf{0 .}$

Proof. Claim $D p_{q} \neq 0$ away from 0 . If not, after a change of coordinates we could suppose $D p_{q}=\mathbf{0}$ along the $x_{n}$ axis. There would be no term in $x_{n}^{q-1} x_{j}$ for any $j=1, \ldots, n$. This proves the claim.

By compactness, for $\|\mathbf{x}\|=1,\left\|D p_{q}(\mathbf{x})\right\| \geqslant k>0$. By homogeneity of degree $q-1$, $\left\|D p_{q}(\mathbf{x})\right\| \geqslant k\|\mathbf{x}\|^{q-1}$ for all $\mathbf{x}$.

Since $D\left(p-p_{q}\right)$ is of degree $\leqslant q-2,\left\|D\left(p-p_{q}\right)(\mathbf{x})\right\| \leqslant M\left(\|\mathbf{x}\|^{q-2}+1\right)$ for some $M$. Then for $\|\mathbf{x}\|$ large, $\|D p(\mathbf{x})\| \geqslant 1$. The result follows by Corollary 1 of Theorem 2 .

For $n=2$, there is a repeated root if and only if after a change of variables

$$
p_{q}(x, y)=x^{2}\left(a_{0} x^{q-2}+\ldots+a_{q-2} y^{q-2}\right)
$$

if and only if with these coordinates $p_{q}(x, y)$ has no term in $y^{q-1} x$ or $y^{q}$.

LEMMA 1. Let $p: \mathbf{R}^{n} \rightarrow \mathbf{R}$ be $C^{1}$ with local minimum at $\mathbf{0}$ and no other critical point. Then $\operatorname{deg}(D p, B, \mathbf{0})=1$ for $B$ a ball centred at $\mathbf{0}$.

Proof. Since 0 is the only critical point it suffices to show $\operatorname{deg}(D p, M, 0)=1$ for $M$ any $n$-manifold with boundary containing 0 . Let $p(0)=0$. On a small 
neighbourhood $U$ of 0 we may suppose $p(\mathbf{x})>0$ since $D p(x) \neq 0$, for $\mathbf{x} \neq 0$. Let the closed ball $B_{r}(0) \subseteq U$ with $p(\mathbf{x}) \geqslant \delta>0$ for $\|x\|=r$. Take $k \in(0, \delta)$ and let $M=\left\{x \in B_{r}(0): p(x) \leqslant k\right\}$. Then $M$ is compact, and $D p$ points outward at all boundary points of $M$. By the Poincaré-Hopf Theorem of Milnor (1965), p. 35, the sum of the indices of $D p$ at its zeros is $\chi(M) . M$ is contractible since any integral curve for $-D p$ must converge to 0 , giving $\chi(M)=1$. (Let $d x / d t=-D p x(t)$, and $x(t(n)) \rightarrow x_{\infty}$ for a sequence $t(n) \rightarrow \infty$. Then $x(t(n))$ is in a neighbourhood of $x_{\infty}$ isomorphic by the implicit function theorem to the product of an open level set for $p$ through $x_{\infty}$, and a real interval corresponding to the value of $p$. This contradicts the fact $p(x(t(n)))$ is decreasing.)

LEMMA 2. Let $p(x, y)$ be a polynomial in two variables, homogeneous of degree $q$, with $n$ linear factors $a_{i} x+b_{i} y$, none repeated. Then for $B$ a ball centred at $\mathbf{0}$, $\operatorname{deg}(D p, B, 0)=1-n$. (There are $\frac{1}{2}(q-n)$ quadratic factors.)

Proof. Suppose $p$ is equal to zero along two rays. As we move anticlockwise through an angle $\theta$ from the first to the second, $D p$ moves clockwise through an angle $\pi / 2-\theta$. Going through all $2 n$ of these ups and downs of $p$, we see that $D p$ winds $n-1$ times clockwise round 0 .

THEOREM 4. Let $p(x, y)$ be a cubic in two variables. Suppose $p$ has a local minimum at 0 . Then $p$ has other critical points.

Proof. If there are no repeated roots in $p_{3}$, the cubic term, Theorem 3 gives the result. Thus we let $p_{3}$ have a double or triple root, and after a linear transformation we have either

(i) $p(x, y)=x^{2} y+a x^{2}+2 b x y+c y^{2}$ or

(ii) $p(x, y)=x^{3}+a x^{2}+2 b x y+c y^{2}$.

For a local minimum at 0 we must have $a>0, b>0, b^{2}<a c$, under the supposition $D p(\mathbf{x}) \neq 0$ for $\mathbf{x} \neq \mathbf{0}$, which gives $p(\mathbf{x})>0$ for $\mathbf{x} \neq \mathbf{0}$ near $\mathbf{0}$.

(i) We want to find $(x, y)$ with

$$
\mathbf{0}=D p(x, y)=\left(2 x y+2 a x+2 b y, 2 b x+2 c y+x^{2}\right) .
$$

From $D_{2} p=0$ we have $y=-x(x+2 b) / 2 c$. And substituting in $D_{1} p=0$ and cancelling $x$ (assuming $x \neq 0$ ) gives $x^{2}+3 b x+2\left(b^{2}-a c\right)=0$. This has real roots, $\neq 0$.

(ii) We want $(x, y)$ with

$$
0=D p(x, y)=\left(3 x^{2}+2 a x+2 b y, 2 b x+2 c y\right) .
$$

We solve $3 x^{2}+2\left(a-b^{2} / c\right) x=0$. This has a nonzero root. 
THEOREM 5. Let $p(x, y)$ be a quartic in two variables. Suppose $p$ has a local minimum at $\mathbf{0}$ and this is the only critical point. Then $p$ has a global minimum at 0.

Proof. We have many cases exhibited below. We leave the reader to check most of the results, which involve calculation of degrees by diagram sketching.

(0) There are no repeated linear factors in $p_{4}$, the homogeneous part of degree 4. $\operatorname{Deg}(D p, B, 0)=-3$ or -1 or $p$ has a minimum at 0 .

(I) There is a fourfold linear factor in $p_{4}$, after a linear transformation,

$$
p(x, y)=\delta x^{4}+d x^{3}+e x^{2} y+f x y^{2}+g y^{3}+a x^{2}+2 b x y+c y^{2} .
$$

(a) $g \neq 0, \operatorname{deg}(D p, B, 0)=0$.

(b) $g=0, f \neq 0$, degree $=-1$.

(c) $g=f=0, p$ has a minimum at 0 .

(II) There is a triple factor in $p_{4}$,

$$
p(x, y)=x^{3} y+d x^{3}+e x^{2} y+f x y^{2}+g y^{3}+a x^{2}+2 b x y+c y^{2} .
$$

(a) $g \neq 0, f \neq 0$, degree $=-1$.

(b) $g \neq 0, f=0$, degree $=-1$

(c) $g=0, f \neq 0$, degree $=-2$.

(d) $g=f=0$, degree $=-1$.

(III) There is a double factor and two other linear factors in $p_{4}$,

$$
p(x, y)=x^{2} y(\delta y+h x)+d x^{3}+e x^{2} y+f x y^{2}+g y^{3}+a x^{2}+2 b x y+c y^{2} .
$$

1. $h \neq 0$

(a) $g \neq 0$, degree $=0,-1$ or -2 .

(b) $g=0$, degree $=-1,-2$ or -3 .

2. $h=0$

(a) $g \neq 0, d \neq 0$, degree $=-1$.

(b) $g \neq 0, d=0$

(i) $\delta=-1$, degree $=0$.

(ii) $\delta=1$

(a) $e^{2}<4 a$, degree $=0$.

(b) $e^{2}>4 a$, degree $=-2$.

(c) $e^{2}=4 a$

( $\alpha)$ ef $\neq 4 b$, degree $=-1$.

( $\beta)$ ef $=4 b$, degree $=-2,-1$ or 0.

(c) $g=0, d \neq 0$, degree $=-2,-1$ or 0 .

(d) $g=d=0$

(i) $\delta=-1$, degree $=-3$.

(ii) $\delta=1$ 
(a) $e^{2}<4 a, f^{2}<4 c$, minimum at 0 .

(b) $e^{2}>4 a, f^{2}>4 c$, degree $=-3$.

(c) $e^{2}>4 a, f^{2}<4 c$ or vice versa, degree $=-1$.

(d) $e^{2}=4 a$

(i) $f^{2}<4 c$

(a) $4 b \neq e f$, degree $=0$.

(b) $4 b=e f$, minimum at 0 .

(ii) $f^{2}>4 c$

(a) $4 b \neq e f$, degree $=-2$.

(b) $4 b=e f, 0$ not the only critical point.

(iii) $f^{2}=4 c$

(a) $4 b \neq e f$, degree $=-1$.

(b) $4 b=e f, 0$ not the only critical point.

(IV) There is a double linear factor and a quadratic,

$$
p(x, y)=\delta x^{2}\left(x^{2}+y^{2}\right)+\ldots .
$$

(a) $g \neq 0$, degree $=0$.

(b) $g=0$

(i) $f^{2}>4 \delta c$, degree $=-1$.

(ii) $f^{2}<4 \delta c$, use Corollary 1 of Theorem 2 .

(iii) $f^{2}=4 \delta c$

(a) $e f \neq 4 b$, degree $=0$.

(b) $e=b=0$, minimum at 0 .

(c) $e f=4 b \neq 0,0$ not the only critical point.

THEOREM 6. Let $p$ be a cubic in $x, y$ and $z$, with local minimum at 0 . Then $p$ has another critical point.

Proof. By Theorem 3, we may assume

$$
\begin{aligned}
p(x, y, z)=a x^{3}+b y^{3} & +c x^{2} y+d x^{2} z+e x y^{2}+f y^{2} z \\
& +g x y z+h x^{2}+i y^{2}+j z^{2}+2 k x y+2 m x z+2 n y z .
\end{aligned}
$$

$D_{3} p=0$ when $z=-\left(d x+f y^{2}+g x y+2 m x+2 n y\right) / 2 j=Q(x, y)$ say. Let

$$
P(x, y)=p(x, y, Q(x, y)) \text {. }
$$

A critical point $(x, y)$ for $P$ gives a critical point $(x, y, Q(x, y))$ for $p$ by the chain rule. Since $P$ is of degree $\leqslant 4$ with local minimum at 0 , we need to show $P(x, y)<0$ for some $(x, y) . \quad P_{4}(x, y)=\left(d x^{2}+f y^{2}+g x y\right)^{2} /-4 j$. If $P(x, y) \geqslant 0$ then $P_{4}=0$. Assuming $P(x, y) \geqslant 0$ still, $P_{3}=0$, which contradicts $p$ being a cubic. 


\section{References}

J. Cronin (1964), Fixed points and topological degree in nonlinear analysis, Math. Surveys, No. 11 (Amer. Math. Soc., Providence, R.I.).

J. W. Milnor (1965), Topology from the differentiable viewpoint (The University Press of Virginia, Charlottesville).

University of Auckland

Auckland, New Zealand 